\title{
UTILIZAÇÃO DE MEDICAMENTOS POR DISCENTES QUE UTILIZAM O SERVIÇO DE ASSISTÊNCIA PSICOSSOCIAL DO INSTITUTO DE CIÊNCIAS DA SAÚDE DA UNIVERSIDADE FEDERAL DO PARÁ
}

Angela Valéria de Araújo Batista ${ }^{1}$, Paulo Henrique Costa Braga ${ }^{1}$, Orenzio Soler ${ }^{1}$

${ }^{1}$ Universidade Federal do Pará E-mail para correspondência: soler@gmail.com Submetido em: 20/04/2020 e aprovado em: 23/06/2020

\section{RESUMO}

Introdução: A saúde mental pode incluir a capacidade de um indivíduo de apreciar a vida e procurar um equilíbrio entre as atividades e os esforços para atingir a resiliência psicológica. Admite-se, entretanto, que o conceito de Saúde Mental é mais amplo que a ausência de transtornos mentais. Objetivos: Conhecer o perfil de utilização de medicamentos em discentes utentes do Serviço de Assistência Psicossocial do Instituto de Ciências da Saúde da Universidade Federal do Pará. Métodos: Estudo transversal, prospectivo, feito por amostragem de conveniência e consecutiva, realizado entre janeiro e junho de 2017, utilizando-se de estratégias aplicadas ao cuidado farmacêutico. Resultados e discussão: Os discentes são predominantemente pardos e morenos, idade média de 24 anos, renda familiar de até 3 salários mínimos e de amplo espectro quanto ao extrato social. Significativo grau de conhecimento de utentes sobre seu tratamento farmacológico e dos medicamentos prescritos. Houve necessidade de intervenção farmacêutica quanto a revisão de estratégias farmacoterapêuticas prescritas e de conciliação de medicamentos. Regular adesão ao tratamento farmacológico. Regular satisfação dos utentes com sua qualidade de vida. Conclusões: Infere-se que utentes com diagnóstico de depressão atendidos no Serviço de Assistência Psicossocial do Instituto de Ciências da Saúde da Universidade Federal do Pará apresentam um perfil regular quanto a qualidade da prescrição e o uso de medicamentos; assim como quanto a sua percepção de qualidade de vida.

Palavras-chave: Psiquiatria, Depressão, Assistência Farmacêutica, Cuidado Farmacêutico. Medicamento.

\begin{abstract}
Introduction: Mental health can include an individual's ability to enjoy life and seek a balance between activities and efforts to achieve psychological resilience. It is admitted, however, that the concept of Mental Health is broader than the absence of mental disorders. Aims: To know
\end{abstract}


the profile of medication use in students who use the Psychosocial Assistance Service of the Institute of Health Sciences of the Federal University of Pará. Methods: Cross-sectional, prospective study, carried out by convenience and consecutive sampling, carried out between January and June 2017, using strategies applied to pharmaceutical care. Results and discussion: The students are predominantly brown and brown, with an average age of 24 years, a family income of up to 3 minimum wages and a broad spectrum of social strata. Significant level of knowledge of users about their pharmacological treatment and prescription drugs. There was a need for pharmaceutical intervention regarding the review of prescribed pharmacotherapeutic strategies and medication reconciliation. Regular adherence to pharmacological treatment. Regular user satisfaction with their quality of life. Conclusions: It is inferred that users diagnosed with depression treated at the Psychosocial Assistance Service of the Institute of Health Sciences of the Federal University of Pará have a regular profile regarding the quality of the prescription and the use of medications; as well as their perception of quality of life. Keywords: Psychiatry, Depression, Pharmaceutical care, Medicine.

\section{INTRODUÇÃO}

Vive-se uma alta incidência de depressão da população mundial. A comunidade científica está cada vez mais longe de um consenso quanto às causas da doença, muito embora determinadas condutas terapêuticas sejam praticamente unanimidade, a exemplo da medicalização. A depressão ocupa na atualidade o $4^{\circ}$ lugar entre as causas de ônus em doenças degenerativas e mortes prematuras ${ }^{(1,2)}$.

As informações divulgadas revelam que os índices da doença são crescentes, tendo aumentado 60\% nos últimos quarenta e cinco anos. Agora em 2020, deve atingir entre 15\% e $20 \%$ da população mundial. A depressão ocupará o $2^{\circ}$ lugar na referida classificação, perdendo apenas para as doenças cardíacas ${ }^{(1,3,4)}$.

O termo depressão era usado, inicialmente, para designar sintomas ou caracterizar estados mentais, sendo que o nome da doença era melancolia, termo cunhado há mais de 25 séculos; que, além de indicar uma das doenças mentais, também correspondia a um tipo de temperamento, um estado emocional baixo, infeliz, desanimado e triste ${ }^{(3,5)}$.

Embora a característica mais típica dos estados depressivos seja a proeminência dos sentimentos de tristeza ou vazio, nem todos os utentes relatam a sensação subjetiva de tristeza. Muitos referem, sobretudo, a perda da capacidade de experimentar prazer nas atividades em 
geral e a redução do interesse pelo ambiente. Frequentemente associa-se à sensação de fadiga ou perda de energia, caracterizada pela queixa de cansaço ${ }^{(3,5)}$.

É difícil a percepção do indivíduo em saber que está doente; ou seja, saber que seu estado e as características que ele apresenta são sinais de que ele realmente necessita de ajuda especializada e tratamento. É comum haver a negação da existência de uma doença. Muito embora tristeza e humor depressivo possam indicar síndrome depressiva subjacente, eles também podem fazer parte da adaptação normal a uma doença que ameace a vida ${ }^{(3,5)}$.

De acordo com Farias $^{(6)}$, as principais classes terapêuticas no campo da saúde mental seguem o seguinte percentual: $28 \%$ dos medicamentos dispensados são antidepressivos, $24 \%$ benzodiazepínicos, $21 \%$ antiepilépticos, $10 \%$ antipsicóticos, $8 \%$ barbitúricos, $6 \%$ analgésico de ação central, $2 \%$ antiparkinsoniano e $1 \%$ inibidor de receptor H1. A depressão será a doença mais comum no mundo em $2030^{(1,3,4)}$. Neste contexto, este trabalho teve como objetivo conhecer o perfil de utilização de medicamentos pelos utentes do Serviço de Assistência Psicossocial (SAPS) do Instituto de Ciências da Saúde (ICS) da Universidade Federal do Pará (UFPA).

\section{MÉTODOS}

Trata-se de um estudo transversal, prospectivo, feito por amostragem de conveniência e consecutiva, realizado entre janeiro e junho de 2017, utilizando-se de estratégias aplicadas ao cuidado farmacêutico, fundamentado na pesquisa-ação ${ }^{(7,8,9)}$. Investigou-se discentes de diferentes cursos de graduação da Universidade Federal do Pará, atendidos pelo Serviço de Assistência Psicossocial aos Discentes (SAPS), os quais estavam diagnosticados e em tratamento para o quadro depressivo.

O Serviço de Assistência Psicossocial aos Discentes (SAPS), localizado na Avenida Generalíssimo Deodoro, $\mathrm{n}^{\circ}$ 01, Umarizal, município de Belém do Pará é vinculado ao Instituto de Ciências da Saúde da UFPA (ICS). Oferece serviços em Psiquiatria, Psicoterapia, Terapia

de Grupo, Terapia de Casal e Terapia Familiar. Os atendimentos são realizados de segunda a sexta, nos turnos da manhã das $8 \mathrm{~h}$ às $12 \mathrm{~h}$, e no turno da tarde das $14 \mathrm{~h}$ às $18 \mathrm{~h}$. Para ter acesso ao serviço o discente pode procurá-lo espontaneamente, ou ser, encaminhada pela Pró-Reitoria de Extensão (PROEX) da UFPA, apresentando documento de identificação e encaminhamento.

Este serviço é composto por um quadro técnico de profissionais, entre eles: um MédicoPsiquiatra, quatro Psicólogos, dois Assistentes Sociais, dois Técnicos Administrativos e quatro estagiários (administração, enfermagem, farmácia e assistente social). Não há profissional 
farmacêutico. A realização deste trabalho utilizou-se da aplicação prática dos conceitos relacionados a assistência farmacêutica, farmácia clínica, cuidado farmacêutico, intervenção farmacêutica, adesão ao tratamento, erros de medicação, conciliação de medicamentos, segurança do utente e qualidade de vida.

Foram utilizados formulários estruturados para as entrevistas e coleta de dados. Buscouse os registros em prontuários para confrontar as informações obtidas quanto a farmacoterapia do utente; em especial sobre o medicamento, concentração, dosagem, indicação clínica, posologia utilizada, via de administração e tempo de uso, permitindo assim, a análise da estratégia terapêutica e a necessidade ou não da conciliação de medicamentos ${ }^{(10,11,12)}$.

O Cuidado farmacêutico é uma prática que integra ações de educação em saúde, que incluem atividades de educação permanente para a equipe de saúde e atividades de promoção à saúde de caráter geral e, ações de promoção do uso racional de medicamentos, com o desenvolvimento de atividades assistenciais e técnico-pedagógicas. A atividade assistencial, praticada nos pontos de atenção, inclui os serviços de clínica farmacêutica, que podem ser ofertados ao usuário de forma individual e/ou em atendimentos compartilhados com outros membros da equipe de saúde ${ }^{(10,11,12)}$.

Para verificar o grau de conhecimento do utente sobre seu tratamento farmacológico, os medicamentos prescritos e a medida de adesão ao tratamento, utilizou-se da Escala Likert convertida em padrão dicotômico. Os resultados foram organizados de forma dicotômica de "sim" e "não", sendo apresentados na forma de síntese narrativa. Para a avaliação da qualidade de vida foi utilizado o Instrumento de Avaliação de Qualidade de Vida da Organização Mundial da Saúde (WHOQOL-100) $)^{(14)}$.

As informações foram armazenadas no Excel $^{\circledR}$ versão 2010 e trabalhados estatisticamente no programa BioEstat ${ }^{\circledR}$ versão 5.0. Foi usado o teste binominal para estimação de parâmetros; teste $t$ de Student para amostras independentes cujas variâncias paramétricas são desconhecidas; teste do Qui-quadrado para as medidas de proporções; e ANOVA para comparação de valores.

Com relação aos aspectos éticos, este trabalho seguiu a Resolução do Conselho Nacional de Saúde $\mathrm{n}^{\circ}$ 466, de 12 de dezembro de $2012^{(15)}$, que regulamenta a pesquisa em seres humanos, sendo submetido à Plataforma Brasil sob CAAE n 61004116.0.0000.0018 e aprovado via Parecer Consubstanciado $\mathrm{n}^{\circ}$ 2.048.142. 


\section{RESULTADOS E DISCUSSÃO}

Estudou-se o perfil de 10 discentes diagnosticados e em tratamento para a depressão. 50\% homens e $50 \%$ mulheres. $40 \%$ provenientes da área das ciências da saúde, 30\% das ciências humanas e sociais, $20 \%$ das ciências exatas e naturais e $10 \%$ das letras e artes. A idade dos utentes variou de 19 a 33 anos, com média de 24,5 anos (desvio padrão = 3,95). Quanto a renda familiar 50\% possuem rendimento de até 3 salários mínimos. Quanto ao extrato social foi identificado $30 \%$ (A), $20 \%$ (B), $10 \%$ (C), 30\% (D) e 10\% (E). $80 \%$ se auto-referiram como da raça parda e morena; $10 \%$ branca e $10 \%$ preta.

Os percentuais de medicamentos prescritos e em uso: clonazepam (70\%), citalopram (30\%), bupropiona (20\%), escitalopram (20\%), risperidona (20\%), sertralina (20\%), duloxetina (10\%), fluvoxamina $(10 \%)$, paroxetina $(10 \%)$, prometazina $(10 \%)$, quetiapina $(10 \%)$ e trazodona (10\%). Houve necessidade de intervenção farmacêutica quanto a erros de prescrição e conciliação de medicamentos, visando minimizar eventos adversos aos medicamentos. $\mathrm{O}$ custo médio de tratamento era de $\mathrm{R} \$ 194,44(\mathrm{R} \$ 37,82$ - R $\$ 667,74)$.

Constatou-se, haver significativo conhecimento dos utentes sobre seu tratamento farmacológico (Quadro 1) e sobre os medicamentos prescritos (Quadro 2). Quanto a adesão ao tratamento, $50 \%$ relataram que por vezes são descuidados com o horário de tomar os medicamentos, $20 \%$ disseram que quase sempre acontece e $20 \%$ que ocorre raramente, sendo que $10 \%$ informou que sempre são descuidados com o horário de tomar os medicamentos.

Quanto a qualidade de vida, $40 \%$ dos utentes avaliaram sua qualidade de vida como intermediária; ou seja, nem ruim nem boa. $30 \%$ relataram ter uma qualidade de vida ruim, $20 \%$ informaram ser boa e, $10 \%$ avaliaram como muito boa (Quadro 3).

QUADRO 1 - Conhecimento de utentes sobre seu tratamento farmacológico

\begin{tabular}{|c|c|c|c|}
\hline $\begin{array}{l}\text { Serviço de Assistência Psicossocial } \\
\text { (SAPS) }\end{array}$ & $\begin{array}{l}\text { Sim } \\
\%\end{array}$ & $\begin{array}{c}\text { Não } \\
\%\end{array}$ & p- valor \\
\hline 1- O usuário foi esclarecido sobre seu diagnóstico? & 60 & 40 & $0.7539 *$ \\
\hline 2- O usuário foi esclarecido sobre seu prognóstico? & 40 & 60 & $0.5275^{*}$ \\
\hline $\begin{array}{l}3 \text { - O usuário foi esclarecido sobre a necessidade de mudanças de hábitos e/ou estilo de } \\
\text { vida? }\end{array}$ & 70 & 30 & $0.3438 *$ \\
\hline $\begin{array}{l}\text { 4- O usuário foi esclarecido sobre como será o seu tratamento farmacológico e não } \\
\text { farmacológico? }\end{array}$ & 70 & 30 & $0.3438 *$ \\
\hline $\begin{array}{l}5 \text { - O usuário foi esclarecido sobre por quanto tempo usar e como usar os medicamentos } \\
\text { prescritos? }\end{array}$ & 70 & 30 & $0.3438 *$ \\
\hline $\begin{array}{l}\text { 6- O usuário foi questionado se faz uso de outros medicamentos e/ou de outros tratamentos } \\
\text { concomitantes? }\end{array}$ & 60 & 40 & $0.7539 *$ \\
\hline 7- O usuário ficou satisfeito com o atendimento e/ou o tratamento estabelecido? & 80 & 20 & 0.1094* \\
\hline 8- O usuário ficou satisfeito com o atendimento e/ou atenção dispensada pela farmácia? & 70 & 30 & $0.3438 *$ \\
\hline Média & 65.00 & 35.00 & - \\
\hline Desvio Padrão & 0.12 & 0.12 & $0.0002 * *$ \\
\hline
\end{tabular}


O formulário empregado mostrou-se estatisticamente significativo com p-valor de 0.0002 . A média das respostas positivas foi equivalente a 65.00 e das negativas 35.00 e o desvio padrão de ambas as variáveis foi de 0.12 .

Fonte: Perfil de utilização de medicamentos em discentes que utilizam o Serviço de Assistência Psicossocial do Instituto de Ciências da Saúde da Universidade Federal do Pará, 2018.

QUADRO 2 - Grau de conhecimento de utentes sobre os medicamentos prescritos

\begin{tabular}{|c|c|c|c|}
\hline $\begin{array}{l}\text { Serviço de Assistência Psicossocial } \\
\text { (SAPS) }\end{array}$ & $\underset{\%}{\mathrm{Sim}}$ & $\begin{array}{c}\text { Não } \\
\%\end{array}$ & p-valor \\
\hline 1 - O usuário foi informado e sabe o diagnóstico da doença que está tratando? & 70 & 30 & $0.3438 *$ \\
\hline $\begin{array}{l}2 \text { - O usuário foi informado e sabe para que serve }(\mathrm{m}) \text { o (s) medicamento (s) que irá utilizar } \\
\text { e/ou utiliza? }\end{array}$ & 100 & 0 & $0.0020 *$ \\
\hline $\begin{array}{l}3 \text { - O usuário foi informado e sabe quais os objetivos (resultados esperados) do tratamento } \\
\text { estabelecido? }\end{array}$ & 80 & 20 & $0.1094 *$ \\
\hline 4 - O usuário foi informado e sabe a (s) dose (s) que deve tomar do (s) medicamentos (s)? & 100 & 0 & $0.0020 *$ \\
\hline 5 - O usuário foi informado e sabe os horários de administração dos medicamentos? & 100 & 0 & $0.0020^{*}$ \\
\hline 6 - O usuário foi informado e sabe o modo correto de tomar os medicamentos? & 90 & 10 & $0.0215^{*}$ \\
\hline $\begin{array}{l}7 \text { - O usuário foi informado e sabe se, irá ou não, precisar que alguém lhe administre } \\
\text { medicação? }\end{array}$ & 50 & 50 & $1.0000^{*}$ \\
\hline $\begin{array}{l}8 \text { - O usuário foi informado e sabe se há qualquer restrição (alimentar, ingerir bebidas } \\
\text { alcoólicas, não dirigir ou usar equipamentos que exigem total atenção e controle, entre } \\
\text { outras) durante o uso dos medicamentos? }\end{array}$ & 80 & 20 & $0.1094 *$ \\
\hline $\begin{array}{l}9 \text { - O usuário foi informado e sabe dizer se os medicamentos podem causar algum efeito } \\
\text { colateral e o que fazer caso isto ocorra? }\end{array}$ & 30 & 70 & $0.2061 *$ \\
\hline $\begin{array}{l}10 \text { - O usuário foi informado e sabe dizer se os medicamentos podem causar alguma reação } \\
\text { adversa e o que fazer caso isto ocorra? }\end{array}$ & 30 & 70 & $0.2061 *$ \\
\hline $\begin{array}{l}11 \text { - O usuário foi informado e sabe dizer o que deve fazer caso se esqueça de tomar o } \\
\text { medicamento na hora marcada? }\end{array}$ & 20 & 80 & $0.0578 *$ \\
\hline $\begin{array}{l}12 \text { - O usuário foi informado e sabe dizer em que casos os medicamentos que usa podem } \\
\text { interferir em exames laboratoriais e o que fazer nesses casos? }\end{array}$ & 0 & 100 & $0.0016^{*}$ \\
\hline 13 - O usuário se sente bem informado sobre o tratamento que lhe foi prescrito? & 70 & 0 & $0.3438^{*}$ \\
\hline 14 - O usuário acredita ser importante estar bem informado sobre o seu tratamento? & 100 & 0 & $0.0020^{*}$ \\
\hline Média & 65.71 & 32.14 & - \\
\hline Desvio padrão & 0.34 & 0.35 & $0.016 * *$ \\
\hline
\end{tabular}

O formulário empregado para avaliar o grau de conhecimento de utentes sobre os medicamentos prescritos, mostrou-se significativo com p-valor de 0.016 . As respostas positivas tiveram média de 65.71 e desvio padrão de 0.34 , em comparação as negativas que tiveram média de 32.14 e desvio padrão de 0.35 .

Fonte: Perfil de utilização de medicamentos em discentes que utilizam o Serviço de Assistência Psicossocial do Instituto de Ciências da Saúde da Universidade Federal do Pará, 2018.

QUADRO 3 - Instrumento de avaliação da qualidade de vida

\begin{tabular}{|c|c|c|c|c|c|c|}
\hline Parâmetros & $\underset{\%}{\mathrm{MR}}$ & $\begin{array}{l}\mathrm{R} \\
\%\end{array}$ & $\begin{array}{l}\mathrm{NR} / \mathrm{NB} \\
\%\end{array}$ & $\begin{array}{l}\mathrm{B} \\
\%\end{array}$ & $\begin{array}{c}\mathrm{MB} \\
\%\end{array}$ & p-Valor \\
\hline 1- Como você avaliaria sua qualidade de vida? & 0 & 30 & 40 & 20 & 10 & $0.0001^{*}$ \\
\hline 2 - Quão satisfeito você está com a sua saúde? & 0 & 20 & 20 & 50 & 10 & $0.0001 *$ \\
\hline Parâmetros & $\begin{array}{l}\mathrm{ND} \\
\%\end{array}$ & $\mathrm{MP}$ & $\begin{array}{c}\mathrm{MM} \\
\%\end{array}$ & $\begin{array}{c}\mathrm{BT} \\
\%\end{array}$ & $\begin{array}{c}\mathrm{EX} \\
\%\end{array}$ & p-Valor \\
\hline $\begin{array}{l}3 \text { - Em que medida você acha que sua dor (física) impede você de } \\
\text { fazer o que você precisa? }\end{array}$ & 70 & 0 & 30 & 0 & 0 & $0.0001 *$ \\
\hline $\begin{array}{l}4 \text { - O quanto você precisa de algum tratamento médico para levar sua } \\
\text { vida diária? }\end{array}$ & 20 & 30 & 20 & 30 & 0 & $0.0001^{*}$ \\
\hline 5 - O quanto você aproveita a vida? & 10 & 20 & 50 & 20 & 0 & $0.0001^{*}$ \\
\hline 6 - Em que medida você acha que sua vida tem sentido? & 20 & 10 & 10 & 50 & 10 & $0.0001 *$ \\
\hline 7 - O quanto você consegue se concentrar? & 0 & 30 & 40 & 20 & 10 & $0.0001 *$ \\
\hline 8 - Quão seguro (a) você se sente em sua vida? & 20 & 30 & 20 & 20 & 10 & $0.0404 *$ \\
\hline 9 - Quão saudável é o seu ambiente físico? & 20 & 10 & 10 & 40 & 20 & $0.0001 *$ \\
\hline 10- Você tem energia suficiente para seu dia a dia? & 0 & 40 & 60 & 0 & 0 & $0.0001 *$ \\
\hline 11 - Você é capaz de aceitar sua aparência física? & 10 & 40 & 40 & 10 & 0 & $0.0001^{*}$ \\
\hline Parâmetros & ND & MP & MD & MT & $\mathrm{C}$ & p-Valor \\
\hline
\end{tabular}




\begin{tabular}{|c|c|c|c|c|c|c|}
\hline & $\%$ & $\%$ & $\%$ & $\%$ & $\%$ & \\
\hline 12-Você tem dinheiro suficiente para satisfazer suas necessidades? & 20 & 40 & 20 & 10 & 10 & $0.0001 *$ \\
\hline $\begin{array}{l}\text { 13- Quão disponíveis para você estão às informações que precisa no } \\
\text { seu dia a dia? }\end{array}$ & 0 & 10 & 60 & 0 & 30 & $0.0001^{*}$ \\
\hline 14 - Em que medida você tem oportunidades de atividades de lazer? & 10 & 30 & 40 & 20 & 0 & $0.0001^{*}$ \\
\hline Parâmetros & $\begin{array}{c}\text { MR } \\
\%\end{array}$ & $\begin{array}{l}\mathrm{R} \\
\%\end{array}$ & $\begin{array}{c}\text { NR/NB } \\
\%\end{array}$ & $\begin{array}{l}\mathrm{B} \\
\%\end{array}$ & $\begin{array}{c}\mathrm{MB} \\
\%\end{array}$ & $\mathrm{p}$-Valor \\
\hline 15 - Quão bem você é capaz de se locomover? & 0 & 10 & 0 & 50 & 40 & $0.0001 *$ \\
\hline Parâmetros & $\begin{array}{c}\mathrm{MI} \\
\%\end{array}$ & $\begin{array}{l}\mathrm{I} \\
\%\end{array}$ & $\begin{array}{c}\mathrm{NS} / \mathrm{NI} \\
\%\end{array}$ & $\begin{array}{l}\mathrm{S} \\
\%\end{array}$ & $\begin{array}{c}\mathrm{MS} \\
\%\end{array}$ & p-Valor \\
\hline 16 - Quão satisfeito (a) você está com seu sono? & 0 & 20 & 30 & 30 & 20 & $0.0001 *$ \\
\hline $\begin{array}{l}17 \text { - Quão satisfeito (a) você está com sua capacidade de desempenhar } \\
\text { atividades do seu dia a dia? }\end{array}$ & 10 & 20 & 30 & 20 & 20 & $0.0404 *$ \\
\hline 18 - Quão satisfeito (a) você está com sua capacidade de trabalho? & 0 & 20 & 30 & 30 & 20 & $0.0001 *$ \\
\hline 19 - Quão satisfeito (a) você está consigo mesmo? & 10 & 30 & 30 & 30 & 0 & $0.0001 *$ \\
\hline 20 - Quão satisfeito (a) você está com suas relações pessoais? & 30 & 0 & 20 & 40 & 10 & $0.0001 *$ \\
\hline 21 - Quão satisfeito (a) você está com sua vida sexual? & 30 & 20 & 30 & 20 & 0 & $0.0001 *$ \\
\hline $\begin{array}{l}22 \text { - Quão satisfeito (a) você está com o apoio que você recebe de seus } \\
\text { amigos? }\end{array}$ & 10 & 20 & 30 & 20 & 20 & $0.0404 *$ \\
\hline $\begin{array}{l}23 \text { - Quão satisfeito (a) você está com as condições do local onde } \\
\text { mora? }\end{array}$ & 10 & 10 & 20 & 30 & 30 & $0.0005^{*}$ \\
\hline $\begin{array}{l}24 \text { - Quão satisfeito (a) você está com o seu acesso aos serviços de } \\
\text { saúde? }\end{array}$ & 10 & 20 & 10 & 30 & 30 & $0.0005^{*}$ \\
\hline 25 - Quão satisfeito (a) você está como seu meio de transporte? & 30 & 20 & 20 & 20 & 10 & $0.0404 *$ \\
\hline Parâmetros & $\begin{array}{l}\mathrm{N} \\
\%\end{array}$ & $\begin{array}{c}\mathrm{AV} \\
\%\end{array}$ & $\begin{array}{l}\mathrm{F} \\
\%\end{array}$ & $\begin{array}{c}\mathrm{MF} \\
\%\end{array}$ & $\begin{array}{l}\mathrm{S} \\
\%\end{array}$ & p-Valor \\
\hline 26 - Com que frequência você tem sentimentos negativos & 0 & 40 & 0 & 40 & 20 & $0.0001 *$ \\
\hline Média & 13.08 & 22.31 & 27.31 & 25.00 & 12.69 & \\
\hline Desvio Padrão & 0.15 & 0.12 & 0.16 & 0.15 & 0.12 & $0.0003 * *$ \\
\hline *Qui-Quadrado; **ANOVA & & & & & & \\
\hline
\end{tabular}

Legenda: Parâmetros: Muito Ruim (MR), Ruim (R), Nem Ruim/Nem Boa (NR/NB), Boa (B), Muito Boa (MB), Nada (ND), Muito Pouco (MP), Mais ou Menos (MM), Bastante (BT), Extremamente (EX), Médio (MD), Muito (MT), Completamente (C), Muito Insatisfeito (MI), Insatisfeito (I), Nem Satisfeito/Nem Insatisfeito (NS/NI), Satisfeito (S), Muito Satisfeito (MS), Nunca (NC), Algumas Vezes (AV), Frequente (F), Muito Frequente (MF), Sempre (S).

Nota: Formulário adaptado de Fleck (2000), mostrando-se estatisticamente significativo com p-valor de 0.0003. A média das colunas 1, 2, 3, 4 e 5 foi $(13.08 ; 22.31 ; 27.31 ; 25.00 ; 12.69$; respectivamente) e desvio padrão $(0.15 ; 0.12 ; 0.16 ; 0.15 ; 0.12$; respectivamente). Segundo a estatística as colunas que tiveram significância entre si foram 1 e 3 ( $p=0.01) ; 1$ e 4 ( $p=0.05) ; 3$ e 5 ( $p=0.01) ; 4$ e 5 ( $\mathrm{p}=0.05)$.

Fonte: Perfil de utilização de medicamentos em discentes que utilizam o Serviço de Assistência Psicossocial do Instituto de Ciências da Saúde da Universidade Federal do Pará, 2018.

Os achados relacionados ao perfil socioeconômico dos discentes que utilizam o Serviço de Assistência Psicossocial (SAPS) estão em consonância com outros estudos atualmente publicados $^{(16,17)}$. A depressão é uma das reações a perdas e a ameaças de perda, seja de emprego ou de um contexto social estruturante, que podem induzir à fragmentação da identidade psíquica $^{(18)}$.

O grau de conhecimento de utentes sobre sua estratégia terapêutica e dos medicamentos prescritos foi significativo. A falta de informação sobre aspectos do tratamento é uma das principais razões para que $30 \%$ a $50 \%$ dos utentes não utilizem os medicamentos adequadamente $^{(19)}$. Ibanez e colaboradores ${ }^{(18)}$ identificaram que o menor grau de conhecimento dos utentes está relacionado com a dose $(51,9 \%)$ e a frequência de administração $(33,4 \%)$ dos medicamentos. 
Observou-se regular adesão ao tratamento; assim, como a necessidade de intervenção farmacêutica quanto a revisão de estratégias farmacoterapêuticas prescritas e da conciliação de medicamentos; sendo todas as proposições de ajustes farmacoterapêuticos acatadas pela equipe multidisciplinar. Borba e colaboradores ${ }^{(16)}$ ao analisarem o total de medicamentos por classe medicamentosa prescritos aos utentes portadores de transtorno mental, constataram predomínio de antipsicóticos e estabilizadores do humor, representados, respectivamente, por 32,3\% e $22,8 \%$. Verificou-se que $39 \%$ dos entrevistados tinham mais de três medicamentos prescritos e que faziam uso de medicação via oral, $52 \%$ precisavam ingerir mais de cinco comprimidos diários para o tratamento do transtorno mental. Outrossim, Borba e colaboradores ${ }^{(17)}$ registraram baixa adesão à terapêutica medicamentosa em pacientes com desordens mentais, sendo as variáveis associadas à adesão foram sexo, renda individual, histórico familiar de transtorno mental, percepção sobre sua saúde, diagnóstico de transtorno mental, tempo de doença e de tratamento, tentativa de suicídio, deixar de tomar o medicamento alguma vez no último mês e participação da família.

A satisfação de utentes com sua qualidade de vida foi regular. A World Health Organization $^{(1)}$ destacam que utentes depressivos exigem mudanças de hábitos e/ou estilo de vida para facilitar sua qualidade de vida, a exemplo da higiene do sono, por meio do estabelecimento de horários regulares de sono e vigília, evitando alimentar-se em excesso, fumar e ou ingerir bebidas alcoólicas antes de dormir, criando um ambiente apropriado para o sono e, se possível, praticar atividade física regular e orientada. Thiengo e colaboradores ${ }^{(21)}$, registram que quanto maior o escore de qualidade de vida nos aspectos físico, psicológico e ambiental, maior a satisfação global com os resultados do tratamento. Ao se trabalhar com medidas de satisfação, podem ocorrer vieses que resultem em respostas excessivamente positivas; tais como a gratidão e/ou aceitação. Em relação a estudos em serviços públicos, é provável que os familiares estivessem relutantes em criticar os serviços dos quais eles são dependentes, tendo o receio em perder o acesso ao serviço.

\section{CONCLUSÕES}

Infere-se que utentes com diagnóstico de depressão atendidos no Serviço de Assistência Psicossocial (SAPS) do Instituto de Ciências da Saúde (ICS) da Universidade Federal do Pará (UFPA) apresentam um perfil regular quanto a qualidade da prescrição e o uso de medicamentos; assim como quanto a sua percepção de qualidade de vida. 


\section{REFERÊNCIAS BIBLIOGRÁFICAS}

1. WHO (World Health Organization). Mental health action plan 2013-2020. WHO Document Production Services, Geneva, Switzerland. 2013. 45p. ISBN 9789241506021. https://apps.who.int/iris/bitstream/handle/10665/89966/9789241506021_eng.pdf

2 Capistrano ABP. Revisão dos mecanismos de monitoramento, avaliação e qualificação da Política Nacional de Saúde Mental, Álcool e outras Drogas a partir dos princípios da reforma psiquiátrica brasileira e da garantia dos direitos humanos. Lisboa. Dissertação (Mestrado) Universidade Nova de Lisboa. Faculdade de Ciências Médicas Políticas e Serviços de Saúde Mental. Portugal; 2016.

3. Franco SM, Costa FZN, Leão ALMS. Depressão: mal do século ou demanda do século? Núcleo de Estudos Organizacionais. Face. UFMG. Belo Horizonte. Farol: Revista de Estudos Organizacionais e Sociedade. 2016;3(6):307-354. ISSN 2358-6311. DOI: https://doi.org/10.25113/farol.v3i6.2722

4. Treichel CAS, Campos RTO, Campos GWS. Impasses e desafios para consolidação e efetividade do apoio matricial em saúde mental no Brasil. Interface (Botucatu). 2019;23:e180617. ISSN 1414-3283. http://dx.doi.org/10.1590/interface.180617

5. Onocko-Campos RT. Saúde mental no Brasil: avanços, retrocessos e desafios. Cad. Saúde Pública. 2019;35(11):e00156119. doi:10.1590/0102-311X00156119

6. Farias JT. Análise da prescrição de psicotrópicos dispensados em um Centro de Atenção Integral à Saúde em João Pessoa, Paraíba. João Pessoa. Graduação (Monografia) Universidade Federal do Paraíba. 2015. https://repositorio.ufpb.br/jspui/handle/123456789/1001

7. Thiollent M. Metodologia da pesquisa-ação. 18. ed. São Paulo: Cortez, 2011. 136p. ISBN: 9788524917165

8. Minayo MCS. Análise qualitativa: teoria, passos e fidedignidade. Ciênc. saúde coletiva [Internet]. 2012;17(3):621-626. https://doi.org/10.1590/S1413-81232012000300007 9. Yin R K. Estudo de caso: planejamento e métodos. 5. ed. Bookman: Porto Alegre, RS, Brasil. 2015. ISBN 978-85-8260-232-4

10. Correr CJ, Otuki M. A prática farmacêutica na farmácia comunitária. Porto Alegre: Artmed, 2013. 454p. ISBN: 9788565852821.

11. CFF (Conselho Federal de Farmácia). Resolução nº 585, 29 de agosto de 2013. Regulamenta as atribuições clínicas do farmacêutico e dá outras providências. Diário Oficial da União. 
Brasília, DF, 29 ago. 2013.

12. BRASIL. Congresso Nacional. Lei $n^{\circ} 13.021$, de 8 de agosto de 2014. Dispõe sobre o exercício e a fiscalização das atividades farmacêuticas e dá outras providências. Diário Oficial da União - Seção 1 - Edição Extra - 11/8/2014, Página 1. Brasília. DF.

13. BRASIL. Ministério da Saúde. Secretaria de Ciência, Tecnologia e Insumos Estratégicos. Departamento de Assistência Farmacêutica e Insumos Estratégicos Capacitação para implantação dos serviços de clínica farmacêutica. Brasília: Ministério da Saúde, 2014. 308 p. il. (Cuidado farmacêutico na atenção básica; caderno 2) ISBN 978-85-334-2198-1

14. Fleck MPA. O instrumento de avaliação de qualidade de vida da Organização Mundial da Saúde (WHOQOL-100): características e perspectivas. Ciência \& Saúde Coletiva. 2000;5(1):33-38. ISSN 1678-4561

15. BRASIL. Conselho Nacional de Saúde. Resolução n ${ }^{\circ} 466$ de 12 de dezembro de 2012. Aprova as diretrizes e normas regulamentadoras de pesquisas envolvendo seres humanos. Brasília, Publicada no DOU no 12 - quinta-feira, 13 de junho de 2013 - Seção 1 - Página 59. 16. Borba LO, Maftum MA, Vayego AS, Kalinke LP, Ferreira ACZ, Capistrano FC. Perfil do portador de transtorno mental em tratamento no CAPS. REME - Rev Min Enferm. 2017;21:e1010. DOI: $10.5935 / 1415-2762.20170020$

17. Borba LO, Maftum MA, Vayego SA, Mantovani MF, Felix JVC, Kalinke LP. Adherence of mental therapy for mental disorder patients to drug health treatment. Rev Esc Enferm (USP). 2018;52:e03341. DOI: http://dx.doi.org/10.1590/S1980-220X201700660334

18. Jardim S. Depressão e trabalho: Ruptura de Laço Social. Revista Brasileira de Saúde Ocupacional. 2011;36:84-92. ISSN 0303-7657

19. Dresch AP, Amador TA, Heineck I. Conhecimento dos usuários sobre medicamentos prescritos por odontólogos no sul do Brasil. Ciência \& Saúde Coletiva. 2016;21(2):475-483. DOI: $10.1590 / 1413-81232015212.17732014$

20. Ibanez G, Mercedes BPC, Vedana KGG, Miasso AI. Adesão e dificuldades relacionadas ao tratamento medicamentoso em pacientes com depressão. Rev. bras. enferm. [Internet]. 2014; 67(4):556-562 https://doi.org/10.1590/0034-7167.2014670409. 
21. Thiengo DL, Fonseca D, Abelha L, Lovisi GM. Satisfação de familiares com o atendimento oferecido por um Centro de Atenção Psicossocial Infanto juvenil (CAPSi) da cidade do Rio de Janeiro. Cad. Saúde Colet. 2015;23(3):298-308. DOI: 10.1590/1414-462X201500030172 